\title{
Performance Improvement in Lifetime and Energy of LEACH Protocol
}

\author{
Raksha Bajpai $^{1}$, Prof. M.A Rizvi ${ }^{2}$
}

Abstract: One of the limitations of sensor nodes (WSN) is the limited energy of nodes. To maximize the lifetime of the sensor node and the network, it is preferable to distribute the energy throughout the network by this it minimize the maintenance and maximize overall network performance. To investigate the mechanism to conserve and balance the energy consumption in wireless sensor network, energy minimization due to the path loss in wireless sensor network. Many techniques have been proposed for fault detection, fault tolerance and repair in sensor networks. Cluster-based algorithm is the way to performs failure detection and energy consumed significantly low energy, it improvises energy and balance the energy consumption in wireless sensor network, but it has some limitation. Here it is suggest redefining the existing LEACH algorithm in such a way it will reduce the energy consumption during the transmission and also compare with existing algorithm.

\section{INTRODUCTION}

In wireless Sensor Network Sensors are capable of monitoring a wide variety of ambient conditions such as temperature, pressure, and motion. Because sensors are powered by batteries, the issue of sensors' energy-efficiency is a main concern and it is a most challenging task for the design of wireless sensor network. Speedily development of embedded computing, wireless communication and micro-sensors gives rise to the wireless sensor network (WSN) in which a lot of low-cost micro-sensors are selforganized sensor through wireless links. A node in the network is no longer useful when its battery dies. The fundamental objectives for WSN are ease of deployment, accuracy, flexibility, cost effectiveness, and, reliability. In wireless sensor network (WSN), the majority energy of nodes is used for data transmission and aggregation. Balance this energy consumption and network lifetime have to choose the good Routing algorithm, so a good routing algorithm must balance the energy consumption of nodes to extend the network life time. Many Routing protocols and algorithms for energy-efficiency have been proposed. The cluster-based hierarchical model is better than the one-hop or multi-hop model, so hierarchical based Routing protocol is better than the Multipath Routing Protocol in case of energy saving. One of the best Hierarchical protocols is Low Energy Adaptive Clustering Hierarchy (LEACH).

The main function of LEACH is that the cluster members $(\mathrm{CN})$ elect cluster head $(\mathrm{CH})$ to avoid excessive energy consumption. But still LEACH have some deficiencies such as 1) cluster member nodes exhaust energy after cluster head was dead.; 2) unreasonable $\mathrm{CH}$ selection while the nodes have different energy; 3) some are very big clusters and some are very small clusters in the network at the same time

${ }^{1}$ Department of Computer Engineering\& Application NITTTR, Bhopal, M.P, India

${ }^{2}$ Department of Computer Engineering\& Application NITTTR, Bhopal, M.P, India 
due to no limitation for the number of members in the cluster means clusters are not balanced. By structure of LEACH, we propose a Redefine algorithm.

\section{LEACH Protocol Description}

LEACH (Low Energy Adaptive Clustering Hierarchy) is a hierarchical-based routing protocol. LEACH is a self adaptive and self organized. All the nodes in a network organize themselves into local clusters, with one node acting as the cluster-head $(\mathrm{CH})$ and others are normal nodes. In this two level are form 1st level is Base station (BS), and 2nd is Cluster Head level $\mathrm{S}(\mathrm{CH})$. Random rotation taken by nodes required to be the cluster-heads $(\mathrm{CH})$ to evenly distribute energy consumption in the network. Then the cluster head aggregates and compresses the information received from all the nodes present in the network and sends it to the base station (BS). Therefore, being a cluster-head $(\mathrm{CH})$ node is much more energyintensive than being a non-cluster-head node in the network.

If cluster-head node dies all the nodes that belong to the cluster lose communication ability, so Cluster Head play the important role in network. LEACH incorporates randomized rotation by this function avoid draining the battery of any one sensor in the network. Cluster-head node knows all the cluster members, CH create a TDMA schedule that tells each node exactly when to transmit its data to Cluster Head, using a TDMA schedule for data transfer prevents intra-cluster collisions in the network. LEACH divided into rounds and each round consists of two phases.

Set-up Phase

Advertisement Phase

(2)Cluster Set-up Phase

Steady Phase

(1) Schedule Creation

(2) Data Transmission

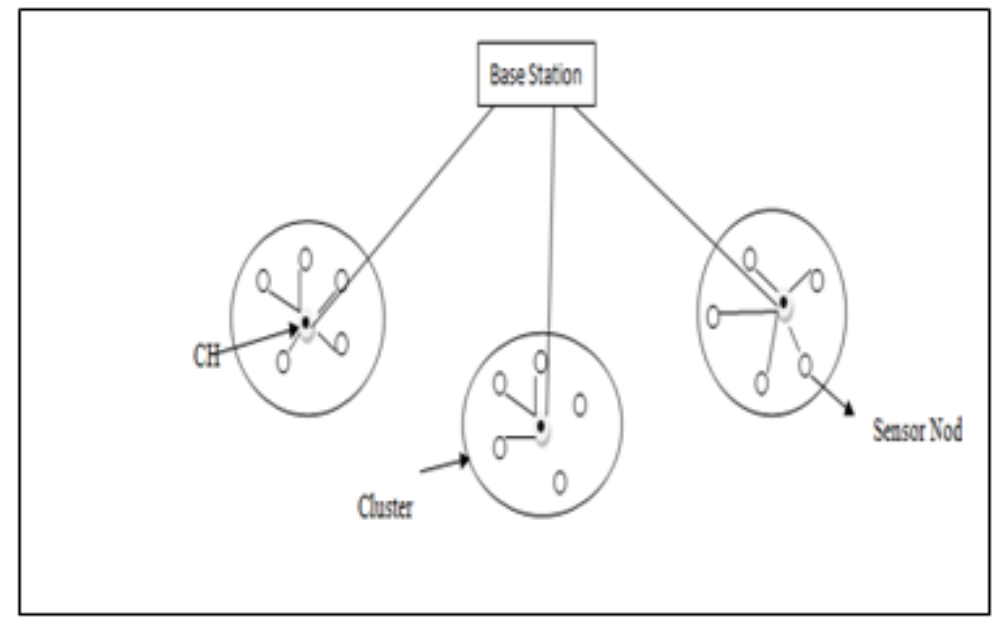

Fig.1. Cluster Formation in LEACH.

\section{Set-up Phase}

During this setup phase, all messages received within the cluster, the $\mathrm{CH}$ creates a TDMA schedule, pick a CSMA code randomly, and broadcast the TDMA table to cluster members every node wanting to be the cluster-head chooses a value, between 0 and 1 , if number is less than a threshold $\mathrm{T}(\mathrm{n})$, the node become a cluster head for the current round. The threshold $T$ (n) value depends upon the desired percentage to 
become a $\mathrm{CH}-\mathrm{p}$, the current round $\mathrm{r}$, and the set of nodes that have not become the cluster-head in the last $1 / \mathrm{p}$ rounds. Elected $\mathrm{CH}$ broadcasts an advertisement message to the rest of the nodes in the network to invite them to join their $\mathrm{CH}$, non-cluster head nodes decide to join the $\mathrm{CH}$ Based upon the strength of the advertisement signal.

\section{Steady Phase}

All cluster-head $(\mathrm{CH})$ waits to receive data from all nodes in its cluster network and then sends the aggregated data or compressed data result back to a Base Station (BS).

The related work of Hierarchical routing summarize in below table. LEACH inspired many Hierarchical routing protocol such as TEEN (Threshold sensitive energy efficient network protocol), PEGASIS (Power efficient gathering in sensor information system)HEED (Hybrid energy efficient distributed clustering).

Table.1.Below table shows the clustering technique in different type of LEACH

\begin{tabular}{|l|l|}
\hline ALGORITHEM & \multicolumn{1}{|c|}{ CLUSTRING SYSTEM } \\
\hline LEACH & Random probabilistic clustering. \\
\hline LEACH-F & Clustering with fixed number of clusters. \\
\hline LEACH-C & $\begin{array}{l}\text { Concertize algorithm clustering algorithm to } \\
\text { produce better cluster }\end{array}$ \\
\hline APTEEN & $\begin{array}{l}\text { New TDMA schedule is introduce to avoid } \\
\text { collision of close by nodes which fall in same } \\
\text { cluster. These nodes sense similar data and try to } \\
\text { send their data simultaneously. }\end{array}$ \\
\hline TEEN & $\begin{array}{l}\text { Total number of transmission is reducing by } \\
\text { allowing the nodes to transmit only when the } \\
\text { sensed value is less then Threshold value. }\end{array}$ \\
\hline PEGASIS & $\begin{array}{l}\text { Each node communicates with close neighbor and } \\
\text { takes turn in transmitting to the base station, it } \\
\text { reduce the amount of energy spent per round. }\end{array}$ \\
\hline
\end{tabular}

\section{Proposed Methodology}

In the proposed methodology an attempt to enhance the energy efficiency is done with initially assuming that all nodes have the same energy and they are static. In this improved methodology of LEACH we take two CHs as compare to older LEACH. At first round find the Primary CH1 from the cluster's sensor node same process do choosing cluster head like earlier in LEACH. Now the same process is follow for choosing secondary $\mathrm{CH} 2$, after the selection of two cluster head, primary $\mathrm{CH} 1$ is $\mathrm{ON}$ mode and secondary $\mathrm{CH} 2$ is on sleep mode. Putting $\mathrm{CH} 2$ on sleep mode because it saves energy. Primary $\mathrm{CH} 1$ do all process done by earlier LEACH after the selection of $\mathrm{CH}$. After the data transmission done by primary 
$\mathrm{CH} 1$ send all data information of all nodes to secondary $\mathrm{CH} 2$. Now the secondary $\mathrm{CH} 2$ take the place of $\mathrm{CH} 1$ and does all .

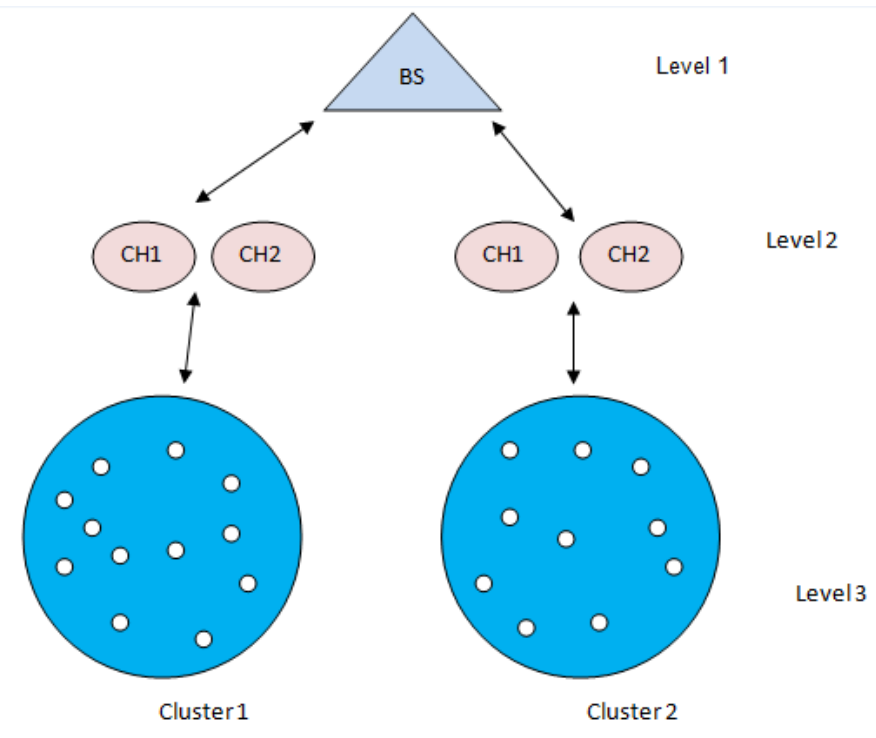

Fig.2.LEACH with two CHs Below improved LEACH algorithm with two CH

\section{Setup Phase}

Assumptions: All nodes are static and the initial energy of all the nodes is same

1) $\mathrm{CN}==\mathrm{r}$

2) If $\mathrm{r}<\mathrm{T}(\mathrm{n})$, then primary $\mathrm{CH}=\mathrm{CN}$ else, go to step 1

3) For secondary $\mathrm{CH}$, if $\mathrm{r}<\mathrm{T}(\mathrm{n})$, then secondary $\mathrm{CH}=\mathrm{CN}$ else, goto step1

4) Primary $\mathrm{CH}==>\mathrm{G}: \mathrm{id}(\mathrm{CH})$,join_adv

5) Secondary $\mathrm{CH}$ sleep mode $\mathrm{ON}$

6) A(i) $\rightarrow$ Primary $\mathrm{CH}(\mathrm{j})$ :id (A(i)),id (Primary $\mathrm{CH}(\mathrm{j})$ ), join_req

7) Primary $\mathrm{CH}(\mathrm{j}) \rightarrow \mathrm{A}(\mathrm{i}): \mathrm{id}$ (Primary $\mathrm{CH}(\mathrm{j})$ ), $<\mathrm{t}(\mathrm{i}), \mathrm{id}(\mathrm{A}(\mathrm{i}))>$

8) After data transmission by Primary $\mathrm{CH}$, now Secondary $\mathrm{CH}$ sleep mode OFF, Primary $\mathrm{CH}$ transfer all node id data information to Secondary $\mathrm{CH}$

9) Secondary $\mathrm{CH}$ follow step 6,7

10) Primary $\mathrm{CH}$ goto step1 for finding new $\mathrm{CH}$.

\section{Steady Phase}

1) A(i) --> Primary $\mathrm{CH}(\mathrm{j}): \mathrm{id}(\mathrm{A}(\mathrm{i})$, id(CH1(j)),info

2) Primary $\mathrm{CH} \rightarrow \mathrm{BS}: \mathrm{id}(\mathrm{CH}), \mathrm{id}(\mathrm{BS})$,aggr_info

3) Primary $\mathrm{CH}$ sleep mode $\mathrm{ON}$

4) Secondary $\mathrm{CH}$ follow same step 1,2 


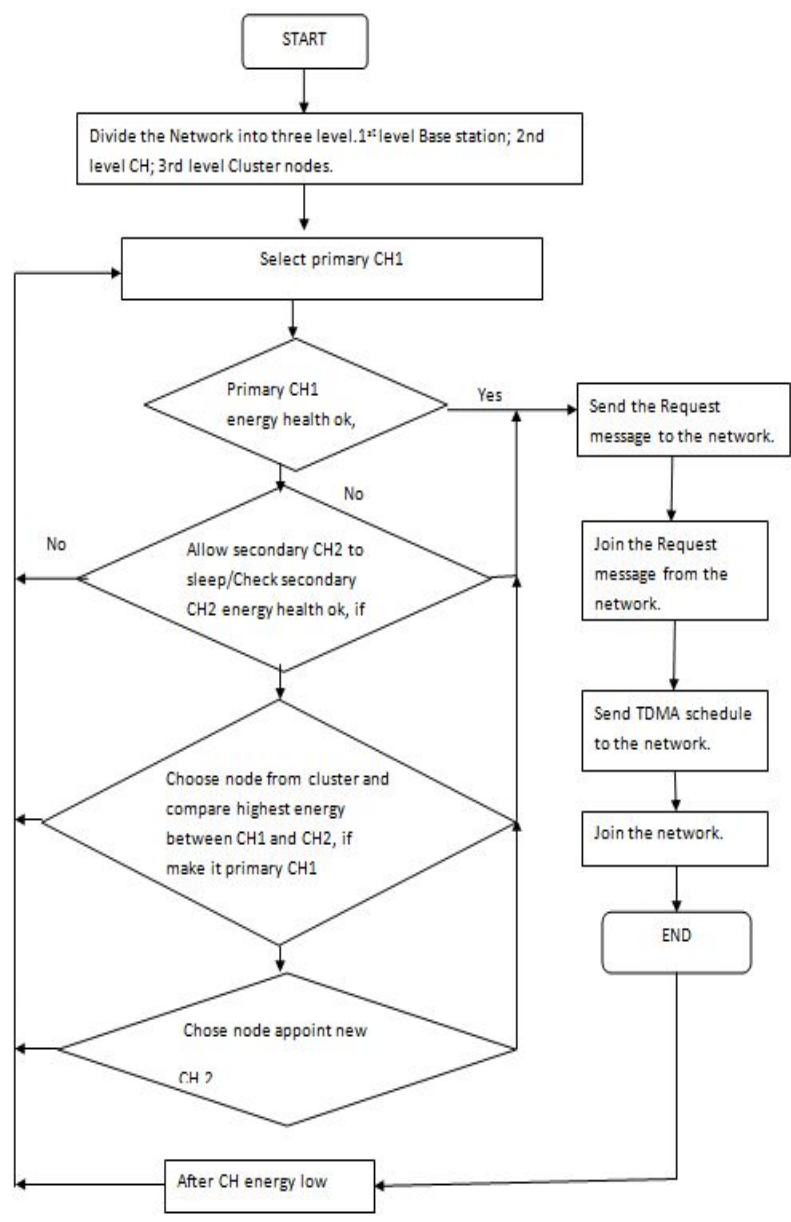

Fig.3.flow chart of Improved LEACH

\section{CONSLUSION}

Based on the hierarchical routing principle of LEACH algorithm, a second new cluster head forming mechanism and data transmission mode is proposed. CHs are uniformly distributed over the network. It is expecting to that the proposed clustering approach of $\mathrm{CH}$ is more energy efficient and continuous data transfer just like LEACH. We propose an extension of LEACH protocol adding two $\mathrm{CH}$ to the respect of $\mathrm{LEACH}$ that considers in second level level of the network. Different types of LEACH protocol commonly use in wireless sensor network we observed that most of them facing problem of network lifetime. So we propose new techniques of energy efficient LEACH protocol for WSN. We will use NS2 (Network Simulator) platform for simulation \& performance analysis for this project. We expect the result comes with reduce the overload from $\mathrm{CH}$ and increases lifetime

\section{REFERENCES}

[1] Chunyao FU1, Zhifang JIANG1, Wei WEI and Ang WEI, “An energy balanced algorithm of LEACH protocol in WSN" IJCSI International Journal of Computer Science Issues, Vol. 10, Issue 1, No 1, January 2013 ISSN (Print): 1694-0784 | ISSN (Online): 1694-0814 www.IJCSI.org.

[2] Lu Tao, Zhu Qing-Xin, Zhang Luqiao," An improvement for LEACH algorithm in wireless sensor network " This work was supported by the National Natural Science Foundation of China (NSFC) under grant No. 60671033 978-1-4244-5046-6/10/\$26.00_c 2010 IEEE. 
[3] V. Loscrì, G. Morabito, S. Marano,"A two-levels hierarchy for low-energy adaptive clustering hierarchy (TL-LEACH)" 0-7803-9152-7/05/\$20.00 (C) 2005 IEEE.

[4] Lejiang Guo, Weijiang Wang, Jian Cui, Lan Gao, " A cluster-based algorithm for energyefficient routing in wireless sensor Networks" 2010 International Forum on Information Technology and Applications, 978-0-7695-4115-0/10 \$26.00 (C) 2010 IEEE DOI 10.1109/IFITA.2010.137.

[5] Wei Liu, LeLe Wang," An improved algorithm based on LEACH protocol" Proceedings of the 2nd International Symposium on Computer, Communication, Control and Automation (ISCCCA-13), Published by Atlantis Press, Paris, France. (C) the authors, 20130464

[6] Qian Liao, Hao Zhu," An energy balanced clustering algorithm based on LEACH protocol" Proceedings of the 2nd International Conference On Systems Engineering and Modeling (ICSEM-13) Published by Atlantis Press, Paris, France. (C) the authors, 20130072

[7] M.Shankar, Dr.M.Sridar, Dr.M.Rajani " Performance evaluation of LEACH protocol in wireless network" International Journal of Scientific \& Engineering Research, Volume 3, Issue 1, January-2012 1 ISSN 2229-5518.

[8] Meena Malik, Dr. Yudhvir Singh , Anshu Arora." Analysis of LEACH protocol in wireless sensor networks" Volume 3, Issue 2, February 2013 ISSN: 2277 128X International Journal of Advanced Research in Computer Science and Software Engineering Research Paper Available online at: www.ijarcsse.com

[9] Seema Bandyopadhyay and Edward J. Coyle," An energy efficient hierarchical clustering algorithm for wireless sensor networks"0-7803-7753-2/03/\$17.00 (C) 2003 IEEE IEEE INFOCOM 2003

[10] Zhen-rui Peng, Hong Yin, Hai-tang Dong and Hui Li," LEACH protocol based two-level clustering algorithm"International Journal of Hybrid Information Technology Vol.8, No.10 (2015), pp.15-26 http://dx.doi.org/10.14257/ijhit.2015.8.10.0 ISSN: 1738-9968 IJHIT Copyright (c) 2015 SERSC 